\title{
Global area strain is a sensitive marker of subendocardial damage in adults after optimal repair of aortic coarctation: three-dimensional speckle-tracking echocardiography data
}

\author{
Ewa Kowalik $^{1} \cdot$ Mirosław Kowalski $^{1} \cdot$ Anna Klisiewicz $^{1} \cdot$ Piotr Hoffman $^{1}$
}

Received: 7 September 2015 / Accepted: 22 January 2016 / Published online: 3 February 2016

(C) The Author(s) 2016. This article is published with open access at Springerlink.com

\begin{abstract}
Aortic coarctation (CoA) in adults is associated with reduced survival. Despite successful repair, some unfavorable changes in the left ventricular (LV) myocardial function are reported. Three-dimensional speckle-tracking imaging (3D-STE) is a novel method that allows to assess regional myocardial function in all directions simultaneously and to calculate global area strain which integrates longitudinal and circumferential deformation. The aim of our study was to assess whether 3-D STE provides any new characteristics of LV deformation in patients with optimal CoA repair. Adults after CoA correction underwent transthoracic echocardiographic examinations. Patients with significant concomitant lesions were ruled out. Global longitudinal strain (GLS), global circumferential strain (GCS), global area strain (GAS), and global radial strain (GRS) were assessed using 3D-STE (Echopac Software, GE). The data were compared with those obtained from healthy subjects. 26 adults (9F/17M; mean age 24.4 years) with repaired CoA were studied. Despite preserved LVEFs, patients with repaired CoA had decreased GAS compared with controls $(-28.8$ vs. $-31.7 \% ; p=0.007)$. No differences between patients and healthy subjects in terms of GLS, GCS and GRS were observed. We found a significant correlation between mean blood pressure and GAS $(R=0.39 ; p<0.05)$. No significant influence of age at repair, CoA correction method or LV mass on three-dimensional deformation was observed. Summarizing, global area strain derived from 3D-STE may be a sensitive indicator of subclinical LV dysfunction in patients after optimal
\end{abstract}

Ewa Kowalik

ewa.kowalik@gmail.com

1 Department of Congenital Heart Disease, Institute of Cardiology, Alpejska 42, 04-628 Warsaw, Poland repair of CoA. Mean blood pressure, but not age at correction seems to determine LV deformation.

Keywords Aortic coarctation - Left ventricular function . Speckle-tracking echocardiography $\cdot$ Three-dimensional imaging · Area strain

\section{Introduction}

Coarctation of the aorta (CoA) is not a localized lesion of the vessel, but a diffused cardiovascular disease. Despite successful surgical or catheter interventional treatment, $\mathrm{CoA}$ is known to be associated with high long-term morbidity and shortened life expectancy [1,2]. In patients treated due to CoA some unfavorable changes in the left ventricular (LV) myocardial function are observed. Data on LV regional function in patients with optimal CoA repair are scarce and limited to two-dimensional imaging $[3,4]$. Three-dimensional speckle-tracking echocardiography (3D-STE) is a novel method that enables quantitative analysis of regional myocardial function of all LV segments in all dimensions simultaneously. The latter technique can also offer a new deformation parameter, area strain, that quantifies endocardial area change and integrates both longitudinal and circumferential deformation [5]. Global area strain (GAS) has been proved to detect early LV systolic dysfunction in athletes, in patients after heart transplantation or after anthracycline therapy, as well as in patients with diabetes and autoimmune disorders [610]. Of note, 3D-STE turned out to be less time consuming than $2 \mathrm{D}$ in respect to the acquisition and analysis time [11, 12], thus the former has a greater chance to be routinely used in the clinical practice. The aim of our study was to assess whether 3D-STE provides any new information on 
myocardial deformation in subjects with optimal repair of CoA.

\section{Methods}

\section{Study population}

We studied 26 consecutive adult patients after surgical or percutaneous CoA correction who were referred to our outpatient clinic for a routine check-up. All patients had preserved LV ejection fraction (EF). The exclusion criteria for the study were: (1) age $>50$ years, (2) CoA repair $<6$ months before the study, (3) recurring or residual $\mathrm{CoA}$ that require intervention, (4) significant concomitant congenital cardiac defects (patients with bicuspid aortic valve were included unless they presented aortic stenosis of any degree and/or more than mild aortic regurgitation), (5) poorly controlled systemic arterial hypertension in follow-up at our center (systolic BP $\geq 140 \mathrm{mmHg}$ and/or diastolic $\mathrm{BP} \geq 90 \mathrm{mmHg}$ ), (6) any evidence of coronary heart disease, (7) arrhythmia at the echocardiographic study, (8) asymptomatic left ventricular dysfunction (LV EF $<55 \%$ ) or congestive heart failure. Echocardiographic data obtained in CoA patients were compared with those of a control group of 18 healthy volunteers who did not have any structural or functional cardiovascular abnormalities, nor did they take any medications. The study was approved by the local ethics committee and each patient signed informed written consent.

\section{Standard echocardiographic imaging and aortic elastic parameters}

Standard transthoracic echocardiographic examinations were performed using commercially available equipment (Vivid 9, GE Vingmed Ultrasound, Horten, Norway) with a matrix probe M3S. Left ventricular end-diastolic (LVEDD) and endsystolic diameters, as well as intraventricular septum (IVS) and posterior wall (PW) thickness were obtained according to ASE guidelines [13]. Relative wall thickness (RWT) was calculated by the formula (2xPW)/LVEDD [14]. Left ventricular ejection fraction (EF) was calculated using the biplane Simpson's method. Left ventricular mass (LVM) was measured according to the Devereux formula [15] and indexed for body surface area. Systolic and diastolic aortic diameters were measured $3 \mathrm{~cm}$ above the aortic valve by 2-D guided M-mode echocardiography at the left parasternal long-axis view: aortic systolic diameter (AoS) at the time of opening of the aortic valve and diastolic diameter (AoD) at the $R$ wave of electrocardiogram. The aortic arch and descending aorta diameters were measured in the suprasternal view. Maximal and mean pressure gradients across the coarctation site were assessed using continuous wave Doppler recordings in the same view. Blood pressure (BP) measurements at the right arm were obtained using a cuff sphygmomanometer (Omron M1, Omron Healthcare Co, Kyoto, Japan). The average of three successive readings was taken into account. Central aortic pulse pressure (PP) was defined as a difference between systolic (SBP) and diastolic blood pressure. Subsequently, we calculated three indices of aortic elastic properties [16]:

Aortic strain $(\%)=100 \times(\mathrm{AoS}-\mathrm{AoD}) / \mathrm{AoD}$

Aortic distensibility $\left(\mathrm{cm}^{2} / \mathrm{dyn} / 10^{6}\right)$

$$
=2 \times(\mathrm{AoS}-\mathrm{AoD}) /(\mathrm{AoD} \times \mathrm{PP})
$$

Aortic stiffness index $=[\ln (\mathrm{SBP} / \mathrm{DBP})] /$

$$
[(\mathrm{AoS}-\mathrm{AoD}) / \mathrm{AoD}]
$$

\section{D-STE analysis}

During the same examination 3D full-volume data sets were acquired from the apical view using the matrix-array transducer 4V-D. Four to six ECG-gated beats were recorded during end-expiratory breath hold to create LV full volume. The volume size and depth were individually adapted and the mean temporal resolution was $25.5 \mathrm{vol} / \mathrm{s}$. 3D data were analyzed offline using EchoPac software (version 113, GE Vingmed Ultrasound). The software automatically detects the $\mathrm{LV}$ endocardial border in 3D, and, after manual adjustment, calculates the LV volumes, cardiac output, stroke volume, EF and LV sphericity index. Next, the definition of the epicardial boundary allows LV mass and 3D global myocardial deformation parameters [longitudinal (GLS), circumferential (GCS), radial (GRS) and area (GAS) strain] to be measured. The area strain is defined as the percentage change in the endocardial area at LV end-systole from its original area at end-diastole [11]. All global strains are weighted averages of regional values from 17 myocardial segments of LV (Fig. 1). Rejected segments (determined automatically by the software) were not taken into account during the calculations of the global strain values and more than three rejected segments in one patient resulted in exclusion from any further analysis. All parameters were compared to those obtained from the healthy subjects in the control group.

\section{Reproducibility}

Intra-observer and inter-observer reproducibility was assessed in 15 randomly chosen subjects. It was expressed as percentage of error, derived as the absolute difference between 2 sets of measurements divided by the mean of the observations and using intraclass correlation coefficients. Intra-observer measures were performed at least 1 month apart. To calculate inter-observer variability the second experienced observer (M.K.), who was blinded to the first observer's findings, analyzed 3D-STE data sets. 


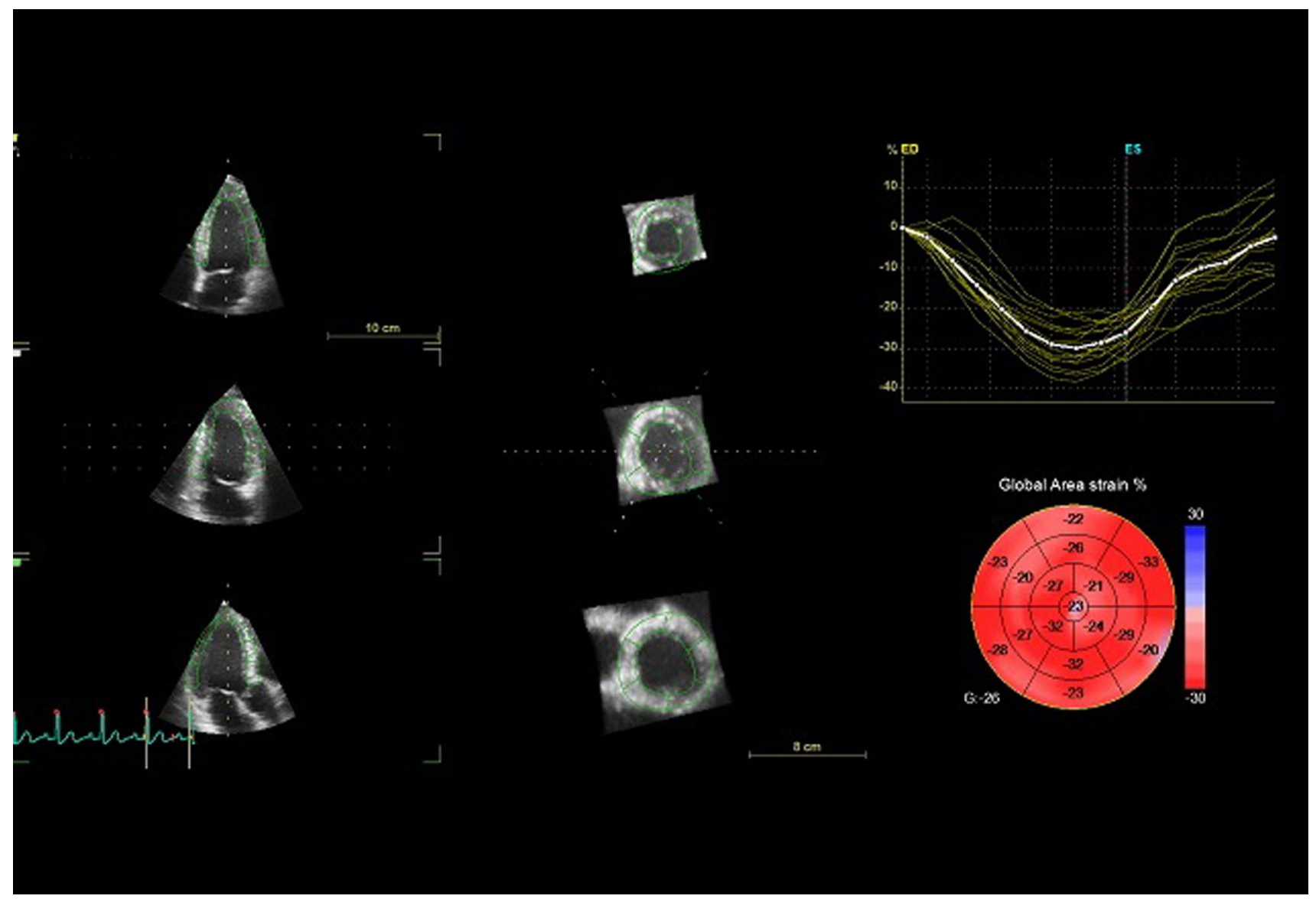

Fig. 1 An example of 3D-STE analysis

\section{Statistics}

Results, unless stated otherwise, are presented as the mean value \pm SD. Normal distribution of variables was checked using the Kolmogorow-Smirnov test. The comparisons of mean differences between the groups were made using unpaired Student's t test and the linear Pearson correlation was used to indicate the strength of a relationship between GAS and other parameters taken into account. In the multivariate regression analysis, the backward elimination method with $\mathrm{t}$ tests was used to select variables. A confidence level of $p<0.05$ was considered statistically significant.

\section{Results}

\section{Clinical data}

Twenty-six patients ( 9 women and 17 men), mean age $24.4 \pm 6.7$ years (range 18-41 years) after surgical $(n=21)$ or percutaneous $(n=5) \mathrm{CoA}$ correction were studied. CoA repair had been performed at a mean age of $8.6 \pm 12.1$ years (range $0-40$ years). In half of the study group bicuspid aortic valve (with no stenosis and only trivial regurgitation) was diagnosed and $58 \%$ of patients were on antihypertensive treatment. No significant differences were observed between patients and controls with respect to age, gender and heart rate. Patients had significantly higher systolic and mean blood pressure. Clinical characteristics of the study group are given in Table 1 .

\section{D echocardiographic data}

Standard echocardiographic parameters in patients and in controls are presented in Table 2. As expected, LV diameters and EF were in normal range and similar between the groups. CoA patients presented an increased LV wall thickness and LV mass comparing to controls. Aortic coarctation was associated with increased aortic stiffness index values. A decrease in aortic distensibility and aortic strain was also observed across the CoA group. 2D echocardiographic examination also revealed that aortic arch and descending aorta were narrower in CoA patients. The mean/peak 
Table 1 Demographics of the study population

\begin{tabular}{|c|c|c|c|}
\hline & Coarctation patients & Healthy controls & $p$ value \\
\hline Mean age (year) & $24.4 \pm 6.7$ & $27.2 \pm 6.7$ & NS \\
\hline $\operatorname{Men}[n(\%)]$ & $17(65)$ & $8(44)$ & NS \\
\hline BMI & $23.8 \pm 3.5$ & $23.5 \pm 2.9$ & NS \\
\hline Heart rate (beats/min) & $59 \pm 10$ & $63 \pm 9$ & NS \\
\hline Systolic blood pressure $(\mathrm{mmHg})$ & $129 \pm 12$ & $119 \pm 9$ & 0.003 \\
\hline Diastolic blood pressure (mmHg) & $80 \pm 10$ & $76 \pm 6$ & NS \\
\hline Mean blood pressure $(\mathrm{mmHg})$ & $96 \pm 10$ & $90 \pm 6$ & 0.016 \\
\hline Bicuspid aortic valve $[n(\%)]$ & $13(50)$ & & \\
\hline Mean age at time of intervention (year) & $8.6 \pm 12.1$ & & \\
\hline Mean time from repair (year) & $15.8 \pm 8.7$ & & \\
\hline \multicolumn{4}{|l|}{ Treatment history } \\
\hline Endovascular stenting $[n(\%)]$ & $5(19)$ & & \\
\hline Prosthetic patch $[n(\%)]$ & $4(15)$ & & \\
\hline End-to-end repair $[n(\%)]$ & $7(27)$ & & \\
\hline Waldhausen operation $[n(\%)]$ & $10(38)$ & & \\
\hline Antihypertensive treatment $[n(\%)]$ & $15(58)$ & & \\
\hline ACEI/ARB $[n(\%)]$ & $9(35)$ & & \\
\hline $\mathrm{CBB}[n(\%)]$ & $11(42)$ & & \\
\hline $\mathrm{BB}[n(\%)]$ & $2(8)$ & & \\
\hline Diuretics $[n(\%)]$ & $3(12)$ & & \\
\hline
\end{tabular}

$B M I$ body mass index, $A C E I$ angiotensin converting enzyme inhibitor, $A R B$ angiotensin receptor blocker, $C B B$ calcium channel blocker, $B B$ beta blocker gradient across coarctation site assessed in patients was $11.2 / 24.4 \mathrm{mmHg}$.

\section{D echocardiographic data}

\section{Feasibility}

Out of all LV segments analyzed $9.1 \%$ were not interpretable and excluded from the analysis of global deformation. The most frequently rejected segments were these located in the posterior wall $(23.5 \%$ of all nonanalyzable segments). An average volume rate for 3D acquisition was $25.5 \pm 9.2 \mathrm{vol} / \mathrm{s}$.

\section{$3 D$ analysis}

3D echocardiographic examination showed that LV volumes and EF were similar in both groups (Table 3). Similarly to the 2D echocardiography, LV mass was significantly higher in patients. Moreover, the 3D LV mass values were higher than the $2 \mathrm{D}$-derived ones, which is consistent with the results of previous studies comparing $2 \mathrm{D}$ and $3 \mathrm{D}$ echocardiographic parameters [17]. All parameters of 3D LV deformation tended to be lower in CoA group, however, only GAS differed significantly in the CoA group. No differences in LV deformation data were found between patients with bicuspid aortic valves compared with tricuspid ones. Nor did the method of CoA correction influenced the LV deformation values. Similarly, there were no differences in 3D deformation values between patients with hypertensive treatment and patients with no medications, although higher LV mass and lower diastolic RR in individuals on antihypertensive medications were observed (Table 4). Both subgroups of patients differ significantly from the control group in terms of GAS values $(p<0.05)$.

Among clinical and echocardiographic parameters characterizing patients with CoA only mean blood pressure correlated significantly with global area strain $(R=0.39$; $p<0.05)$, Fig. 2. Neither age at correction nor LV mass nor aortic elasticity indices correlated with the degree of LV deformation assessed by 3D-STE. Similarly, the multivariate regression showed that mean BP was the only parameter independently associated with LV GAS (cumulative $\left.R^{2}=0.62 ; p=0.002\right)$.

\section{Reproducibility}

Intra- and inter-observer variability for global area strain was $5.4 \%$ (ICCs 0.884) and $9 \%$ (ICCs 0.641). The values are comparable to those reported for GAS in normal adults [11] and patients with other heart diseases [18]. Bland-Altman plots of the GAS intra- and inter-observer differences are presented in Figs. 3, 4. 
Table 2 Standard echocardiographic parameters

\begin{tabular}{lccl}
\hline & Coarctation patients & Healthy controls & $p$ value \\
\hline IVS (mm) & $9.8 \pm 1.6$ & $8.7 \pm 1.6$ & 0.02 \\
PW (mm) & $9.9 \pm 1.2$ & $8.2 \pm 0.9$ & $<0.001$ \\
LVED (mm) & $50.2 \pm 5.1$ & $50.5 \pm 3.6$ & NS \\
LVSD (mm) & $29.9 \pm 4.3$ & $31.4 \pm 3.4$ & NS \\
EF (\%) & $66 \pm 4.7$ & $66.9 \pm 5.2$ & NS \\
RWT & $0.4 \pm 0.06$ & $0.33 \pm 0.04$ & $<0.001$ \\
LVM (g) & $183.1 \pm 52.1$ & $151.8 \pm 35.7$ & 0.02 \\
LVM index & $98.4 \pm 23.2$ & $81.1 \pm 13.3$ & 0.003 \\
MAPSE (mm) & $14.5 \pm 1.6$ & $16.1 \pm 1.9$ & 0.01 \\
E/A ratio & $1.86 \pm 0.73$ & $1.85 \pm 0.73$ & NS \\
Aortic elastic properties & & & \\
Aortic systolic diameter (mm) & $30.4 \pm 7.5$ & $27.6 \pm 3.0$ & NS \\
Aortic diastolic diameter (mm) & $27.8 \pm 7.6$ & $24.4 \pm 3.2$ & 0.03 \\
Aortic strain (\%) & $10.2 \pm 5.7$ & $14.3 \pm 6.7$ & 0.04 \\
Aortic distensibility & $3.2 \pm 1.6$ & $6.4 \pm 3.6$ & 0.003 \\
Aortic stiffness index & $7.1 \pm 5.4$ & $4.0 \pm 2.8$ & 0.02 \\
Aortic arch (mm) & $21.0 \pm 3.8$ & $23.0 \pm 2.1$ & 0.04 \\
Descending aorta (mm) & $14.4 \pm 2.4$ & $17.3 \pm 2.1$ & $<0.001$ \\
Mean gradient across coarctation site (mmHg) & $11.2 \pm 8$ & & \\
Peak gradient across coarctation site (mmHg) & $28.4 \pm 14$ & & \\
\hline
\end{tabular}

IVS interventricular septum, $P W$ posterior wall, $L V E D$ left ventricular end-diastolic diameter, $L V S D$ left ventricular systolic diameter, $E F$ ejection fraction, $R W T$ relative wall thickness, $L V M$ left ventricular mass
Table 3 Real-time three-dimensional echocardiographic assessment

\begin{tabular}{lccl}
\hline & Coarctation patients & Healthy controls & $p$ value \\
\hline LV EDV (mL) & $107.8 \pm 33.6$ & $113.5 \pm 23.3$ & NS \\
LV ESV (mL) & $41.3 \pm 17.6$ & $44.2 \pm 12.0$ & NS \\
SV (mL) & $65.8 \pm 17.4$ & $69.4 \pm 13.6$ & NS \\
CO (L/min) & $4.13 \pm 1.4$ & $4.68 \pm 1.3$ & NS \\
LV EF (\%) & $61.8 \pm 6.9$ & $61.4 \pm 4.8$ & NS \\
Sphericity index & $0.45 \pm 0.09$ & $0.44 \pm 0.09$ & NS \\
LVM (g) & $137.6 \pm 22.4$ & $124.3 \pm 16.5$ & 0.02 \\
LVM index & $75.423 \pm 11.9$ & $67.944 \pm 8.6$ & 0.01 \\
GLS (\%) & $-16.6 \pm 3.8$ & $-18.4 \pm 2.5$ & NS \\
GCS (\%) & $-16.7 \pm 2.8$ & $-17.5 \pm 2.4$ & NS \\
GAS (\%) & $-28.8 \pm 4.1$ & $-31.7 \pm 2.7$ & 0.007 \\
GRS $(\%)$ & $47.1 \pm 10.1$ & $51.3 \pm 6.5$ & NS \\
\hline
\end{tabular}

$E D V$ end-diastolic volume, $E S V$ end-systolic volume, $S V$ stroke volume, $C O$ cardiac output, $L V M$ left ventricular mass, GLS global longitudinal strain, $G C S$ global circumferential strain, $G A S$ global area strain, GRS global radial strain

\section{Discussion}

It was the first study that examined 3D LV deformation from echocardiography in adults after optimal CoA repair. The important new finding is the subtle LV myocardial dysfunction as assessed by global area strain. Area strain reflects the change in the endocardial surface from its original dimensions at end-diastole and it integrates both longitudinal and circumferential deformations of the LV. We speculate that reduced GAS values can reflect subclinical microvascular abnormalities in the population analyzed. In the present study the remaining deformation parameters (longitudinal, circumferential and radial strain) did not differ significantly, which corresponds with optimal clinical outcome of CoA repair. Our results are in agreement with those by Cook et al. who studied a similar group of adults with repaired CoA. Using non-invasive angiography in combination with CMR the authors demonstrated subendocardial perfusion abnormalities despite a lack of LV hypertrophy, epicardial coronary artery disease or recoarctation [19]. Thus, it seems that the novel 3D deformation parameter, global area strain, might become an early non-invasive indicator of subclinical endocardial dysfunction. In contrast, Kutty et al. [20] using CMR based feature-tracking showed no differences between the COA group with normal LV mass and normal controls in terms of GLS and GCS. However, lower GRS values were observed in the patients group. In our opinion these results should be interpreted with caution due to the significant difference in the mean age of compared groups (37.1 years in controls vs. 23.3 years in CoA patients with normal LV mass, $p$ value not given) as the LV deformation values proved to be agerelated [21, 22]. 
Table 4 Selected clinical parameters and echocardiographic indices in coarctation patients with no hypertension and in coarctation patients on antihypertensive medications

\begin{tabular}{lccc}
\hline & CoA pts with no hypertension $(n=11)$ & CoA pts on antihypertensive medications $(n=15)$ & $p$ value \\
\hline Mean age (year) & $25.5 \pm 6.4$ & $23.5 \pm 7.0$ & NS \\
Systolic blood pressure (mmHg) & $129.7 \pm 11.4$ & $128.3 . \pm 12.3$ & NS \\
Diastolic blood pressure (mmHg) & $84.8 \pm 7.5$ & $76.3 \pm 10.1$ & 0.01 \\
LVM index & $87.5 \pm 14.5$ & $106.4 \pm 43.8$ & 0.01 \\
GLS (\%) & $-16.0 \pm 4.4$ & $-17.0 \pm 3.4$ & NS \\
GCS (\%) & $-16.0 \pm 1.6$ & $-17.2 \pm 3.4$ & NS \\
GAS (\%) & $-27.9 \pm 4.0$ & $-29.4 \pm 4.1$ & NS \\
GRS (\%) & $44.9 \pm 8.8$ & $48.7 \pm 11.0$ & NS \\
\hline
\end{tabular}

$C o A$ aortic coarctation, $L V M$ left ventricular mass, GLS global longitudinal strain, GCS global circumferential strain, GAS global area strain, GRS global radial strain

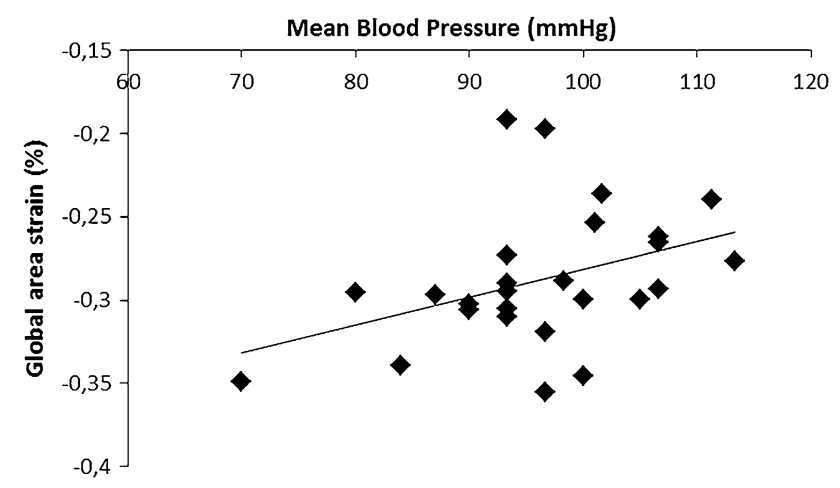

Fig. 2 The correlation between mean BP and GAS in CoA patients $(r=0.39 ; p<0.05)$

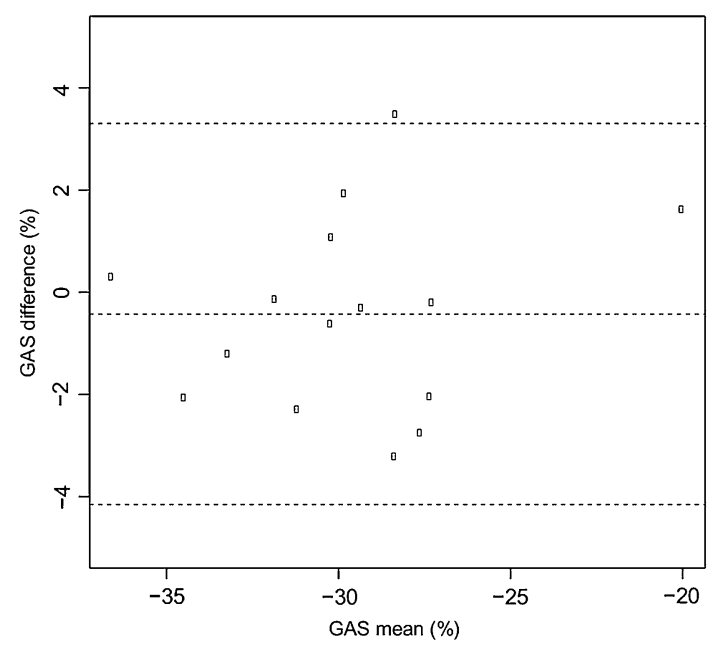

Fig. 3 Bland-Altman plot for intra-observer difference of the global area strain

The subendocardial layer of the myocardial fibers is more vulnerable to functional impairment due to direct impact of intraventricular blood pressure and the

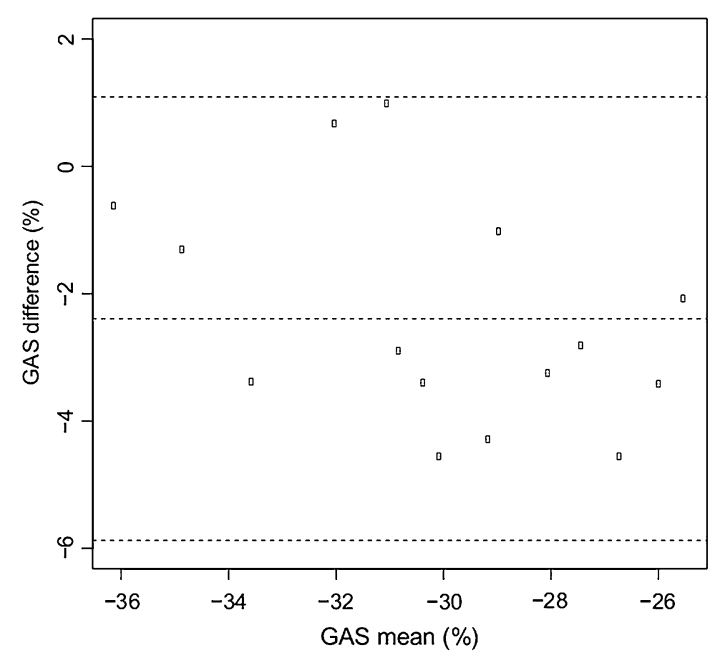

Fig. 4 Bland-Altman plot for inter-observer difference of the global area strain

unfavorable coronary flow in this area. Our analysis showed that the reduction of GAS was associated with an increased pressure overload. The results are consistent in this field with our previous observation that a higher degree of narrowing across the coarctation site leads to greater reductions in local 2D deformation indices for the midsegment of the LV anterior wall [4]. Similar findings regarding the association between GAS and mean BP have also been reported previously in subjects with high-normal blood pressure [12] and native hypertensive patients [23]. However, we observed decreased global area strain values in patients after CoA repair on hypertensive treatment as well as in patients with no hypertension. Thus, the changes found in GAS cannot be explained only by the presence of hypertension.

In contrast to previous data on patients with aortic valvular disease [24] or hypertension [23], no association 
between global deformation parameters and the degree of LV hypertrophy (LVM or RWT) were found in the present study. However, the analyzed population included patients with only slightly increased LV mass and no clear-cut LV hypertrophy.

The age at correction did not have an impact on the values of 3D deformation indices in patients with optimal CoA repair, neither was such a relationship found in our previous study in adults where 2D deformation indices were used [4]. On the other hand, Di Salvo et al. [3] showed a significant correlation between $2 \mathrm{D}$ strain rate and age at correction in children after successful CoA correction. This difference might result from a much younger population analyzed earlier after repair in their study.

Our findings regarding aortic elastic properties are consistent with those reported in previous studies, which showed that aortic strain, distensibility and stiffness index of the ascending aorta remained abnormal despite successful CoA repair [16, 25]. However, the aortopathy did not go along with a significant correlation between these parameters and indices of 3D LV deformation. It is noteworthy that Di Salvo et al. [3] confirmed the relationship between 2D deformation (strain rate) and aortic stiffness index in children after CoA repair. The possible explanation might be a different study population as mentioned above. In addition, the method of deformation analysis (2D longitudinal strain rate derived from septum and LV lateral wall) could impact the results.

Summarizing, our study provides new insights into the LV regional function in patients after optimal CoA repair and the potential explanation of higher rate of cardiac events and reduced life expectancy in this population. The decreased GAS might be an early indicator of late cardiovascular complications and 3D-STE seems to be useful in risk stratification. Thus, further studies will help to define the clinical benefit of 3D deformation analysis.

\section{Limitations}

The major limitation of our study relates to the small sample size mainly due to restrictive inclusion criteria as we decided to assess patients after optimal CoA repair with no significant comorbidities. Different techniques of CoA correction should also be pointed out. Another issue corresponds to the influence of LV imaging quality on deformation analysis. The feasibility of 3D-STE was high in the present study, however, our patients were relatively young and they presented no substantial myocardial hypertrophy. In the study we analyzed only global deformation parameters. The analysis of regional function of $17 \mathrm{LV}$ segments might provide more information, however, such an approach is much more time consuming and hard to apply in every day practice. Finally, the limitations of the 3D-STE analyses are different vendor's algorithms with poor inter-vendor reproducibility [26] that makes the results of the studies difficult to compare.

\section{Conclusions}

Global area strain derived from three-dimensional speckletracking echocardiography detects LV myocardial damage in a subclinical stage in patients after optimal repair of CoA. Mean blood pressure, but not the age at correction or techniques of CoA repair impacts LV deformation.

Acknowledgments Financial support for the study was provided by the Polish Ministry of Science and Higher Education for the statutory activities of the Institute of Cardiology (2.33/V/12).

\section{Compliance with ethical standards}

Conflict of interest The authors declare that they have no conflict of interest.

Open Access This article is distributed under the terms of the Creative Commons Attribution 4.0 International License (http://creativecommons.org/licenses/by/4.0/), which permits unrestricted use, distribution, and reproduction in any medium, provided you give appropriate credit to the original author(s) and the source, provide a link to the Creative Commons license, and indicate if changes were made.

\section{References}

1. Celermajer DS, Greaves K (2002) Survivors of coarctation repair: fixed but not cured. Heart 88:113-114

2. Pedersen TA, Munk K, Andersen NH, Lundorf E, Pedersen EB, Hjortdal VE, Emmertsen K (2011) High long-term morbidity in repaired aortic coarctation: weak association with residual arch obstruction. Congenit Heart Dis 6:573-582

3. Di Salvo G, Pacileo G, Limongelli G, Verrengia M, Rea A, Santoro G, Gala S, Castaldi B, D'Andrea A, Caso P, Giovanna Russo M, Calabro R (2007) Abnormal regional myocardial deformation properties and increased aortic stiffness in normotensive patients with aortic coarctation despite successful correction: an ABPM, standard echocardiography and strain rate imaging study. Clin Sci 113:259-266

4. Kowalski M, Kowalik E, Kotliński K, Szymański P, Kuśmierczyk M, Rózański J, Hoffman P (2009) Regional left ventricular myocardial shortening in normotensive patients late after aortic coarctation repair: normal or impaired? Ultrasound Med Biol 35:1947-1952

5. Kleijn SA, Aly MF, Terwee CB, van Rossum AC, Kamp O (2011) Three-dimensional speckle tracking echocardiography for automatic assessment of global and regional left ventricular function based on area strain. J Am Soc Echocardiogr 24:314-321

6. Liu HY, Deng YB, Liu K, Li Y, Tang QY, Wei X, Chang S, Lu X (2013) Left ventricular systolic strain of the cardiac allograft evaluated with three-dimensional speckle tracking echocardiography. J Huazhong Univ Sci Technol Med Sci 33:765-769

7. Miyoshi T, Tanaka H, Kaneko A, Tatsumi K, Matsumoto K, Minami H, Kawai H, Hirata KI (2014) Left ventricular 
endocardial dysfunction in patients with preserved ejection fraction after receiving anthracycline. Echocardiography 31:848-857

8. Zhang X, Wei X, Liang Y, Liu M, Li C, Tang H (2013) Differential changes of left ventricular myocardial deformation in diabetic patients with controlled and uncontrolled blood glucose: a three-dimensional speckle-tracking echocardiography-based study. J Am Soc Echocardiogr 26:499-506

9. Huang BT, Yao HM, Huang H (2014) Left ventricular remodeling and dysfunction in systemic lupus erythematosus: a three-dimensional speckle tracking study. Echocardiography 31:1085-1094

10. Monte IP, Mangiafico S, Buccheri S, Bottari VE, Lavanco V, Arcidiacono AA, Leggio S, Deste W, Tamburino C (2015) Myocardial deformational adaptations to different forms of training: a real-time three-dimensional speckle tracking echocardiographic study. Heart Vessels 30:386-395

11. Saito K, Okura H, Watanabe N, Hayashida A, Obase K, Imai K, Maehama T, Kawamoto T, Neishi Y, Yoshida K (2009) Comprehensive evaluation of left ventricular strain using speckle tracking echocardiography in normal adults: comparison of three-dimensional and two-dimensional approaches. J Am Soc Echocardiogr 22:1025-1030

12. Tadic M, Majstorovic A, Pencic B, Ivanovic B, Neskovic A, Badano L, Stanisavljevic D, Scepanovic R, Stevanovic P, Celic V (2014) The impact of high-normal blood pressure on left ventricular mechanics: a three-dimensional and speckle tracking echocardiography study. Int J Cardiovasc Imaging 30:699-711

13. Lang RM, Bierig M, Devereux RB, Flachskampf FA, Foster E, Pellikka PA, Picard MH, Roman MJ, Seward J, Shanewise J, Solomon S, Spencer KT, St John Sutton M, Stewart W, American Society of Echocardiography's Nomenclature and Standards Committee, Task Force on Chamber Quantification, American College of Cardiology Echocardiography Committee, American Heart Association, European Association of Echocardiography, European Society of Cardiology (2006) Recommendations for chamber quantification. Eur J Echocardiogr 7:79-108

14. Galiuto L, Badano L, Fox K, Sicari R, Zamorano JL (2011) The EAE textbook of echocardiography. Oxford University Press, Oxford, $\mathrm{p} 28$

15. Devereux RB, Reichek N (1977) Echocardiographic determination of left ventricular mass in man: anatomic validation of the method. Circulation 55:613-618

16. Vogt M, Kühn A, Baumgartner D, Baumgartner C, Busch R, Kostolny M, Hess J (2005) Impaired elastic properties of the ascending aorta in newborns before and early after successful coarctation repair: proof of a systemic vascular disease of the prestenotic arteries? Circulation 111:3269-3273
17. Abramov D, Helmke S, Lel-K R, King DL, Maurer MS (2010) Overestimation of left ventricular mass and misclassification of ventricular geometry in heart failure patients by two-dimensional echocardiography in comparison with three-dimensional echocardiography. Echocardiography 27:223-229

18. Altman M, Bergerot C, Aussoleil A, Davidsen ES, Sibellas F, Ovize M, Bonnefoy-Cudraz E, Thibault H, Derumeaux G (2014) Assessment of left ventricular systolic function by deformation imaging derived from speckle tracking: a comparison between $2 \mathrm{D}$ and $3 \mathrm{D}$ echo modalities. Eur Heart $\mathbf{J}$ Cardiovasc Imaging 15:316-323

19. Cook SC, Ferketich AK, Raman SV (2009) Myocardial ischemia in asymptomatic adults with repaired aortic coarctation. Int $\mathrm{J}$ Cardiol 133:95-101

20. Kutty S, Rangamani S, Venkataraman J, Li L, Schuster A, Fletcher SE, Danford DA, Beerbaum P (2013) Reduced global longitudinal and radial strain with normal left ventricular ejection fraction late after effective repair of aortic coarctation: a CMR feature tracking study. Int J Cardiovasc Imaging 29:141-150

21. Cheng S, Fernandes VR, Bluemke DA, McClelland RL, Kronmal RA, Lima JA (2009) Age-related left ventricular remodeling and associated risk for cardiovascular outcomes: the multi-ethnic study of atherosclerosis. Circ Cardiovasc Imaging 2:191-198

22. Sun JP, Lam YY, Wu CQ, Yang XS, Guo R, Kwong JS, Merlino JD, Yu CM (2013) Effect of age and gender on left ventricular rotation and twist in a large group of normal adults: a multicenter study. Int J Cardiol 167:2215-2221

23. Galderisi M, Esposito R, Schiano-Lomoriello V, Santoro A, Ippolito R, Schiattarella P, Strazzullo P, de Simone G (2012) Correlates of global area strain in native hypertensive patients: a three-dimensional speckle-tracking echocardiography study. Eur Heart J Cardiovasc Imaging 13:730-738

24. Li CM, Li C, Bai WJ, Zhang XL, Tang H, Qing Z, Li R (2013) Value of three-dimensional speckle-tracking in detecting left ventricular dysfunction in patients with aortic valvular diseases. J Am Soc Echocardiogr 26:1245-1252

25. Lam YY, Mullen MJ, Kaya MG, Gatzoulis MA, Li W, Henein MY (2010) Left ventricular and ascending aortic function after stenting of native coarctation of aorta. Am J Cardiol 105:1343-1347

26. Badano LP, Cucchini U, Muraru D, Al Nono O, Sarais C, Iliceto S (2013) Use of three-dimensional speckle tracking to assess left ventricular myocardial mechanics: inter-vendor consistency and reproducibility of strain measurements. Eur Heart J Cardiovasc Imaging 14:285-293 